\title{
Optimization of the formulation and mechanical properties of starch based partially degradable bone cements
}

\author{
LUCIANO F. BOESEL ${ }^{1,2}$, JOÃO F. MANO ${ }^{1,2}$, RUI L. REIS ${ }^{1,2}$ \\ ${ }^{1}$ Department of Polymer Engineering, University of Minho, Campus of Azurém, 4800-058, \\ Guimarães, Portugal \\ ${ }^{2} 3 B^{\prime}$ 's Research Group - Biomaterials, Biodegradables and Biomimetics, University of Minho, \\ Campus of Gualtar, 4710-057, Braga, Portugal
}

\begin{abstract}
Previous studies have shown the possibility of developing in-situ polymerizable hydrogels that disclose a range of properties that might allow for their use as bone cements. Their main advantage is to be partially degradable, which is important to allow bone ingrowth (better fixation). In addition, their uptake of water makes them less agressive for the tissues, disclosing better fatigue properties and ideal for release of drugs when in service.This work reports a statistical study of the formulation of partially degradable acrylic bone cements that include on their composition corn starch/cellulose acetate blends (SCA). The aim was to optimize a set of properties (mechanical, swelling/degradation and curing) by changing the values of some parameters such as SCA amount and particle size and molar ratio of the acrylic monomers. Statistical tests demonstrated that the most important parameter was the molar ratio of monomers, with the SCA percentage also playing a role. It was possible to develop formulations with mechanical properties in the range of ASTM specifications and with polymerization temperatures lower than those of commercial acrylic cements. Some formulations were subsequently selected for tensile and dynamic mechanical thermal analysis (DMA) tests, under dry and wet conditions.
\end{abstract}

(C) 2004 Kluwer Academic Publishers

\section{Introduction}

Since bone cements were first used to fix a prosthesis to the bone in the 1960s, a great deal of effort has gone into designing better formulations that avoid some of the problems posed by conventional acrylic bone cements. Those problems include thermal and chemical necrosis of the bone, prosthesis loosening due to inadequate mechanical and/or biological properties, tissue inflammation, etc. Among the alternatives tried to improve the characteristics of bone cements, one can find the incorporation of bioactive fillers to improve the mechanical properties and increase the adhesion to bone [1], the development of novel formulations with higher ductility and lower modulus [2] to produce a more even stress distribution between the prosthesis and the bone, the use of adhesive additives to increase adhesion between bone and the metal prosthesis and minimize the gap between the interfaces [3] and the modification of the liquid phase in order to decrease the thermal and chemical damage to tissue [4].

An interesting approach would be to combine some of these alternatives with the use of a biodegradable polymer. Such systems, combined with bioactive fillers as the solid component of the bone cement formulation, could induce new bone growth (due to the presence of bioactive particles) not only on the interface cementbone but also in the volume left empty by the degradable polymer as it was being removed. Additionally, the partial substitution of a more hydrophilic and adhesive monomer for methylmethacrylate (MMA) could improve even further the initial adhesion to bone and the growth of new bone inside the material, since this is largely dependent on the water uptake capability of the material. This higher hydrophilicity would favor the release of incorporated drugs (antibiotics) since many of them are water soluble and could then be more easily (and completely) released, avoiding the need of high loadings of drugs that happens with conventional bone cements.

Suitable materials for this objective are starch based blends, already studied for a range of biomedical applications such as scaffolds for tissue engineering [57], systems for drug delivery [8,9] and proposed for bone replacement and regeneration applications $[10,11]$. Pereira et al. [12] used SCA as the solid component of novel bone cement formulations that also incorporated acrylic acid (AA) in order to improve the water uptake and generate $\mathrm{pH}$ sensitivity. These cements presented mechanical properties in the range of those of a polymethylmethacrylate (PMMA) bone cement and a diffusion mechanism adequate for the controlled release 
of drugs or bioactive agents. In a later study, Espigares $e t$ al. [13] incorporated a biocompatible and osteoconductive mineral (hydroxyapatite, HA) to confer a bonebonding character to the cements. These cements were composed by a solid phase (SCA + HA) and a liquid phase (MMA + AA) as traditional PMMA bone cements. Considering the kinetics of curing, mechanical properties and bioactivity, the best results were obtained with a solid/liquid ratio of 55/45 and 20\% of HA.

This paper presents a statistical study of the formulations of starch incorporating bone cements. The study was performed in order to better understand the effect of several factors (namely the starch content and particle size and the monomers ratio) on the mechanical, curing and swelling/degradation properties of the formulations, allowing for the selection of the best conditions for further studies (incorporation of bioactive particles and/or drugs, substitution of other biodegradable materials for SCA, etc).

\section{Materials and methods}

\subsection{Preparation of bone cements}

Specimens were prepared by adding the solid component to the liquid component. The solid was constituted by a commercial corn starch/cellulose acetate 50/50 wt \% blend (SCA), obtained from Novamont, Italy. The liquid was constituted by acrylic acid (AA, Merck, Germany) and methylmethacrylate (MMA, Merck, Germany), which were used as received. Benzoyl peroxide powder (BPO, Merck, Germany) was used as the polymerization initiator at a molar concentration of 0.01 with respect to the monomers amount. BPO was purified by fractional recrystallization from ethanol and subsequently vacuum dried (m.p. $104^{\circ} \mathrm{C}$ ). N-dimethylaminobenzyl alcohol (DMOH) was used as the activator of the initiator, at a molar concentration of 0.67 with respect to the BPO amount.

For studying the effects of three factors (SCA particle size, SCA amount and MMA/AA molar ratio), a design of experiments methodology with a $2^{3}$ full factorial was employed. In this kind of design, three parameters can be studied at two different levels, with all possible combinations among them being tested, what gives a total of eight runs. It then allows for the determination of the effect of each parameter in the desired property, as well as the effect of each second-order interaction (interaction between two factors) and the effect of the third-order interaction (i.e. between the three factors). Detailed explanation about design of experiments and their analysis can be found elsewhere [14, 15]. The levels of each factor were as shown in Table I.

The analysis of the effect of each factor, and their significance, was performed with the "Statistica" ${ }^{\circledR} 5.0$

T A B L E I Levels of each studied factor

\begin{tabular}{lcc}
\hline \multirow{2}{*}{ Factors } & \multicolumn{2}{c}{ Levels } \\
\cline { 2 - 3 } & $(-)$ & $(+)$ \\
\hline SCA particle size $\mu \mathrm{m}$ & $<90$ & $90-125$ \\
SCA\% & 33.2 & 55 \\
MMA/AA & 0.5 & 2.0 \\
\hline
\end{tabular}

T A B L E I I Factors and their levels studied in the second factorial

\begin{tabular}{lcc}
\hline \multirow{2}{*}{ Factors } & \multicolumn{2}{c}{ Levels } \\
\cline { 2 - 3 } & $(-)$ & $(+)$ \\
\hline SCA particle size $\mu \mathrm{m}$ & $<125$ & $250-355$ \\
MMA/AA & 2.0 & 4.0 \\
\hline
\end{tabular}

(StatSoft, USA) software for statistical analysis. The values of the effects were plotted on normal probability plots. In these plots, all of the effects that lie along a line passing through the point $(0,50)$ are negligible, whereas the large effects are far from this line and can then be easily identified $[14,15]$.

A second matrix was then designed, this time a $2^{2}$ one. As shown in Table II. This matrix originates four runs with all possible combinations. The analysis of the effect of each factor, and their significance, was performed with the same software, this time by employing a two-way analysis of variance (ANOVA). An F test, at a 95\% confidence level, was applied to test the significance of each effect $[14,15]$.

The 12 different compositions produced (eight for the first matrix and four for the second) with their respective combination of levels are shown in Tables III and V. The level codification employed is related to the levels of the factors used in each desing and which are shown in Tables I and II. Just as an example, the level codification for composition A was $(+-+)$. The first sign $(+)$ refers to the SCA particle size $(90-125 \mu \mathrm{m}$, in this example); the second sign ( - ) refers to the SCA amount (33.2\%); the third sign ( - ) refers to the MMA/AA molar ratio (0.5). All other formulations in Table III follow the same correspondence. Formulations in Table V are related to the levels shown in Table II; in this case the first sign refers to the level of the SCA particle size while the second sign refers to the MMA/AA molar ratio. As an example, formulation I (codification - + ) contained SCA with particle sizes lower than $125 \mu \mathrm{m}$ and a MMA/ AA ratio of 4.0.

The preparation of specimens for subsequent tests was carried out following the traditional method. The activator DMOH was dissolved in the liquid phase. Initiator of free radical polymerization reaction was added to the solid phase. Both phases were mixed and stirred by hand until the mixture became paste like with a high viscosity; the mass was then placed into a poly(tetrafluoroethylene) (PTFE) mold to allow it to cure. After $60 \mathrm{~min}$, one group of specimens was removed from the mold and put into oven an at $60^{\circ} \mathrm{C}$ until they attained constant weight. Other group was stored at $23^{\circ} \mathrm{C}$ and $55 \% \mathrm{RH}$.

A commercial bone cement (Palacos ${ }^{\circledR}$ R, Schering Plough, Belgium) was prepared following the manufacturer's instructions. This was used as a control for comparative purposes.

\subsection{Curing parameters}

Setting time $\left(t_{\mathrm{s}}\right)$ and maximum polymerization temperature $\left(T_{\max }\right)$ were measured using a PTFE mold (diameter of $40 \mathrm{~mm}$ ) and a thermocouple connected to a digital thermometer with a precision of $\pm 1{ }^{\circ} \mathrm{C}$. The apparatus 
was placed in a room at $18 \pm 1{ }^{\circ} \mathrm{C}$. The powder and liquid components of bone cements were mixed and packed into the PTFE mold when the mixture reached the dough state; the mold was closed with a plate containing a small hole in the center for the thermocouple. The thermocouple was located in the center of the curing mass. Setting time was calculated as the time at which the temperature of the mass was the sum of the room temperature $\left(18^{\circ} \mathrm{C}\right)$ and maximum temperature $\left(T_{\max }\right)$ divided by two.

\subsection{Mechanical properties}

Tensile and compressive tests were carried out at room temperature on an Instron 4505 Universal Mechanical Testing Machine using a load cell of $50 \mathrm{kN}$. A minimum of five specimens were tested for each type of solicitation and each sample. The specimens were tested either after storage at $23^{\circ} \mathrm{C}$ and $55 \% \mathrm{RH}$ or after seven days of immersion in an isotonic saline solution (ISS: $0.154 \mathrm{M}$ $\mathrm{NaCl}$ aqueous solution at $\mathrm{pH} 7.4)$ maintained at $37^{\circ} \mathrm{C}$. In this case, they were tested immediately after being removed from the solution.

For tensile characterization, crosshead speeds of $5 \mathrm{~mm} / \mathrm{min}$ until $1 \%$ strain (for determining the modulus with higher precision) and then of $50 \mathrm{~mm} / \mathrm{min}$ until fracture were employed. A resistive extensometer (10 mm gauge length) was used to measure the strain during the initial part of the test (low crosshead speed). Average dimension of rectangular specimens for tensile tests was $3 \times 5 \times 30 \mathrm{~mm}^{3}$. Fracture surfaces were observed by scanning electron microscopy (SEM) in a Leica Cambridge S360 microscope.

Specimens for compressive were cylinders with $6 \mathrm{~mm}$ diameter and $12 \mathrm{~mm}$ height. The crosshead speed was $20 \mathrm{~mm} / \mathrm{min}$. Tests were carried out up to failure or until $60 \%$ reduction in specimen height.

The mechanical tests with the samples in continuous immersion and the dynamic mechanical thermal analysis (DMA) experiments in dry conditions were carried out in a DMA7e Perkin-Elmer apparatus, using the 3-point bending mode. Bending platforms of 15 and $20 \mathrm{~mm}$ were used, for experiments in wet and dry conditions, respectively.

For the tests in immersion (carried out at $37^{\circ} \mathrm{C}$ ), a metallic reservoir was fitted in the furnace of the equipment. The reservoir was filled with isotonic saline solution. Specimens had cross-section dimensions of $1.5 \times 4 \mathrm{~mm}^{2}$ and were kept one day in the ISS at $37^{\circ} \mathrm{C}$. In the creep tests the creep stress was $1 \mathrm{MPa}$. A program of creep/recovery was used, where the strain, $\varepsilon$, was measured against time.

DMA experiments were performed in the immersed samples. The real (storage modulus, $E^{\prime}$ ), and the imaginary (loss modulus, $E^{\prime \prime}$ ) components of the complex modulus, $E^{*}=E^{\prime}+\mathrm{i} E^{\prime \prime}$, were recorded against frequency, that varied between 0.5 and $10 \mathrm{~Hz}$. A static stress of $0.12 \mathrm{MPa}$ and a dynamic stress of $0.1 \mathrm{MPa}$ were used in such experiments. The DMA experiments on dry samples were performed at constant frequency of $1 \mathrm{~Hz}$, while the temperature was scanned between -10 to $100{ }^{\circ} \mathrm{C}$, at $3^{\circ} \mathrm{C} \mathrm{min}^{-1}$. A static stress of $0.36 \mathrm{MPa}$ and a dynamic stress of $0.3 \mathrm{MPa}$ were used in this case. Specimens had cross-section dimensions of $1.5 \times 4 \mathrm{~mm}^{2}$.

\subsection{Hydration degree and degradation behavior}

The water-uptake and the degradation of the prepared bone cement formulations were studied over a period of 60 days. Specimens (identical to the ones used for the compression tests) were conditioned to constant weight in an oven at $60^{\circ} \mathrm{C}$, before being immersed in $5 \mathrm{~mL}$ of ISS. The specimens were removed at regular intervals of $3,7,14,30$ and 60 days, being taken out of the solution, rinsed with distilled water, blotted on filter paper to remove surface solution/water and immediately weighed. They were then dried in the oven at $60^{\circ} \mathrm{C}$ to constant weight in order to determine the weight loss. Water uptake (WU) was calculated using the following equation:

$$
\mathrm{WU}_{t}=\left[\left(m_{t}-m_{0}\right) / m_{0}\right] * 100
$$

where $m_{t}$ is the mass of the specimen at time $t$ (days) and $m_{0}$ is the mass prior to immersion $(t=0)$. Weight loss (WL) was calculated as follows:

$$
\mathrm{WL}_{t}=\left[\left(m_{f, t}-m_{0}\right) / m_{0}\right] * 100
$$

where $m_{\mathrm{f}, t}$ is the final mass of the specimen kept in the oven until constant mass after $t$ days of immersion in the ISS.

\section{Results and discussion}

\subsection{First matrix}

Table III shows the properties measured for the eight formulations of the first factorial design, together with the levels for each one of the three studied factors. Figs. 1,2 and 3 show the swelling/degradation, mechanical and curing properties, respectively, of some selected formulations. For the sample geometry used in this study, two behaviors could be observed. The compositions containing the highest amount of AA (e.g. B and F in Fig. 1(a) went to equilibrium of water uptake very quickly due to the high hydrophilicity of this monomer, allowing water to rapidly fill all the available sorption sites; after 3 days no appreciable changes on water uptake by these samples were seen. On the other hand, the compositions with lower amount of AA presented a much slower approach to equilibrium, due to the increased difficulty for reaching the hydrophilic sites of the materials. After 14 days there was not yet an indication of equilibrium; although the values for water uptake were approaching those for the previous samples (with high amounts of AA), they were still lower. So, the amount of AA had effects both on the magnitude of sorbed water and on the kinetics of sorption.

This behavior directly correlates with the weight loss (Fig. 1(b)), since the increased water content would allow for more material (plasticizer, low molecular weight molecules,) to be leached out during the initial degradation stage. Despite the equilibrium in WU of samples B and F, the weight decreased continuously, as that water would continue promoting the leaching of 

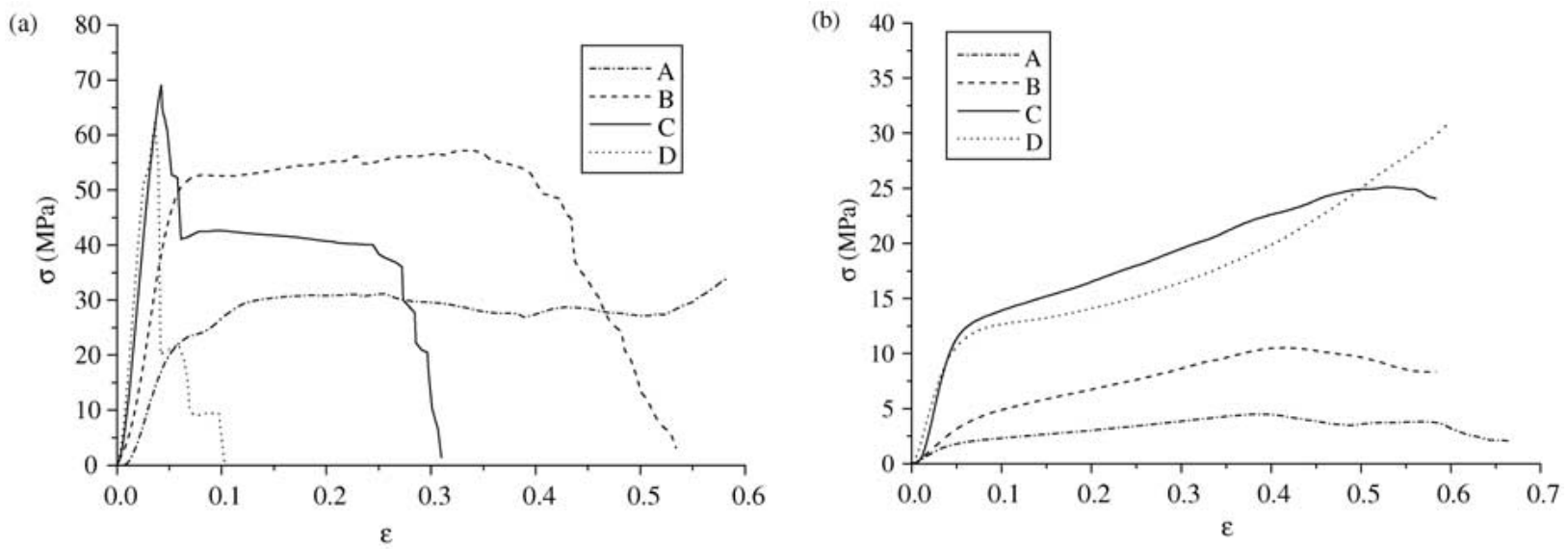

Figure 1 Compressive stress-strain curves of selected specimens tested (a) before and (b) after 7 days of swelling in ISS. Formulations codes are indicated in Table III.
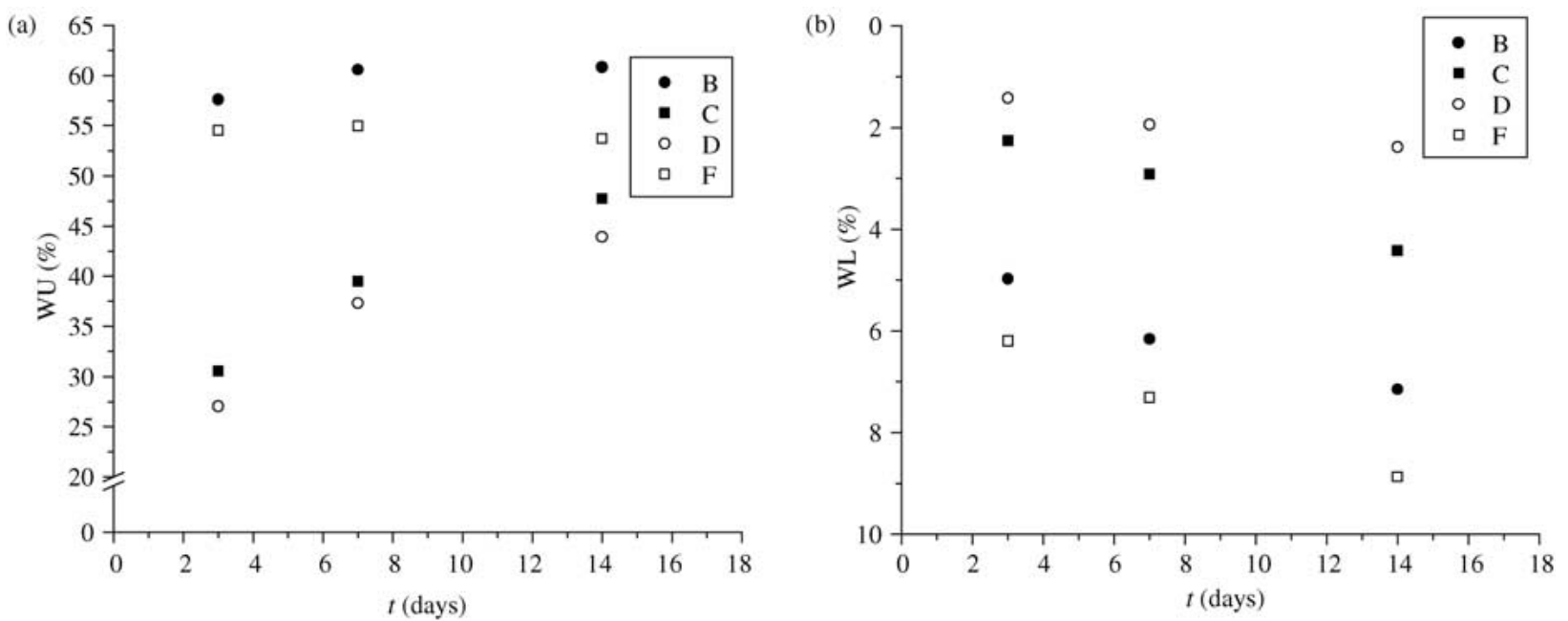

Figure 2 Swelling (a) and degradation (b) of selected samples from the first matrix. Formulations codes are indicated in Table III.

molecules and, later, would cause the degradation of SCA molecules. SCA, being an hydrophilic polymer, also had some effects on these properties, although they were much less relevant when compared to the monomers ratio effect.

Regarding the curing parameters (Table III and Fig. 2), three very different behaviors can be observed: (1) formulations with very high $\left(>120^{\circ} \mathrm{C}\right) T_{\max }$ and very short $t_{\mathrm{s}}$ (B and $\mathrm{F}$ ); (2) formulations with high $T_{\max }$ $\left( \pm 100{ }^{\circ} \mathrm{C}\right.$ ) and long $t_{\mathrm{s}}(\mathrm{D}$ and $\mathrm{G})$; (3) formulations with low $T_{\max }\left(<80^{\circ} \mathrm{C}\right)$ and short $t_{\mathrm{s}}(\mathrm{C}$ and $\mathrm{E})$. The first behavior can be clearly attributed to the higher amounts of AA incorporated in the liquid component, since this monomer is more reactive and has a higher heat of polymerization than MMA [16]. On the other hand, compositions containing more MMA were also influenced by the amount of SCA; the higher it was, the lower the $T_{\max }$ (since there was less monomer to polymerize) and the shorter the $t_{\mathrm{s}}$ (due to the high viscosity). This effect of SCA amount was masked by the high reactivity of $\mathrm{AA}$ in the formulations containing higher levels of this monomer, especially in the case of the $T_{\max }$; only a small shift to shorter times could be detected when the amount of SCA was higher.

The mechanical (compressive) properties of these samples were also very dependent on the composition.

T A B L E I I I Formulations studied and their properties

\begin{tabular}{|c|c|c|c|c|c|c|c|c|}
\hline Formulation & Levels & $\mathrm{WU}_{7}(\%)$ & $\mathrm{WL}_{7}(\%)$ & $\sigma_{\mathrm{y}}(\mathrm{MPa})$ & $E_{1 \%}(\mathrm{GPa})$ & $\sigma_{\text {yas }}(\mathrm{MPa})$ & $T_{\max }\left({ }^{\circ} \mathrm{C}\right)$ & $t_{\mathrm{s}}(\min )$ \\
\hline A & +-- & 54.4 & 7.0 & $23 \pm 5.1$ & $0.66 \pm 0.18$ & $1.4 \pm 0.30$ & 124 & 3.4 \\
\hline B & ++- & 60.6 & 6.2 & $55 \pm 7.4$ & $1.0 \pm 0.10$ & $2.9 \pm 0.19$ & 136 & 2.5 \\
\hline $\mathrm{C}$ & +++ & 39.5 & 2.9 & $70 \pm 11$ & $2.1 \pm 0.18$ & $9.9 \pm 0.42$ & 67.5 & 5.9 \\
\hline $\mathrm{D}$ & +-+ & 37.3 & 1.9 & $26 \pm 9.9$ & $2.1 \pm 0.30$ & $9.5 \pm 0.98$ & 104 & 9.0 \\
\hline $\mathrm{E}$ & -++ & 40.6 & 2.3 & $53 \pm 6.4$ & $1.9 \pm 0.27$ & $9.5 \pm 0.41$ & 76.5 & 6.2 \\
\hline $\mathrm{F}$ & --- & 55.0 & 7.3 & $24 \pm 6.7$ & $0.97 \pm 0.20$ & $1.5 \pm 0.14$ & 149 & 3.6 \\
\hline G & -+- & 59.4 & 6.1 & $22 \pm 5.3$ & $1.7 \pm 0.095$ & $3.0 \pm 0.46$ & 126 & 2.8 \\
\hline $\mathrm{H}$ & --+ & 37.2 & 1.9 & $25 \pm 4.7$ & $2.3 \pm 0.44$ & $9.7 \pm 1.0$ & 116 & 9.6 \\
\hline
\end{tabular}

$\mathrm{WU}_{7}=$ water uptake (after seven days in the saline solution); $\mathrm{WL}_{7}=$ weight loss (after 7 days in the saline solution); $\sigma_{\mathrm{y}}=$ yield compressive strength; $E_{1 \%}=$ secant modulus at $1 \%$ strain; $\sigma_{\text {yas }}=\sigma_{\mathrm{y}}$ after 7 days swelling; $T_{\max }=$ maximum polmerization temperaturey; $t_{\mathrm{s}}=$ setting time. 


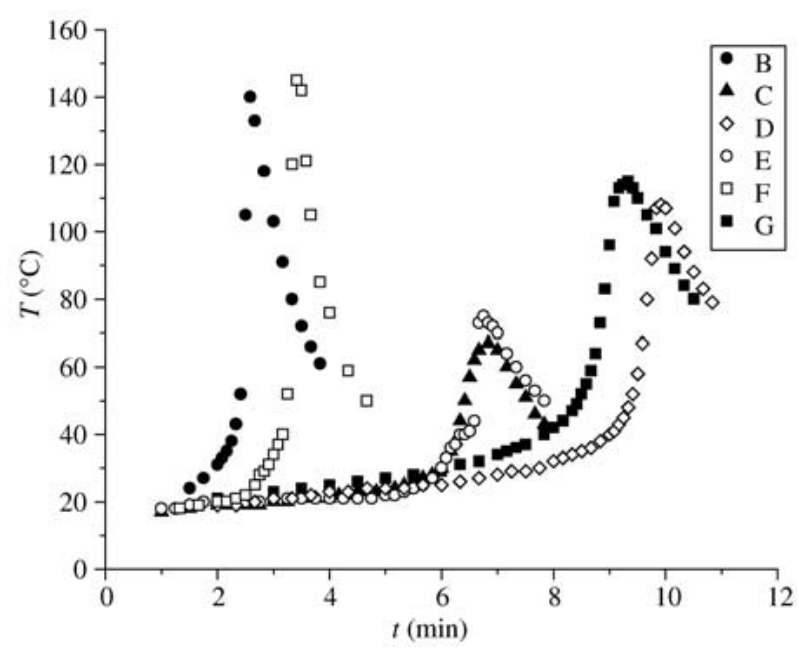

Figure 3 Curing curves of selected specimens. Formulations codes are indicated in Table V.

Fig. 3 shows the stress-strain curves of some selected specimens. Formulations with higher amounts of MMA (C and D) show a very stiff and brittle behavior, typical of acrylic polymers, while those with higher amount of AA (A and B) show a soft and more ductile behavior. As AA is much more hydrophobic than MMA, samples with higher amounts of the former would absorb more water during the storage in the $55 \% \mathrm{RH}$ chamber; this water would act as a plasticizer for the SCA and/or acrylic, decreasing the mechanical strength and increasing the ductility of the formulations. As it is shown in Table III, all formulations with the low level of the third factor had much lower $E_{1 \%}$; than the ones with the higher level. There is the exception of formulations E and G, which disclose almost the same modulus. The SCA amount had not a well-defined effect; sometimes it influenced mainly the $\sigma_{y}$ (compare, e.g. $C \times D$ ), while other times the effect was on the modulus $(\mathrm{F} \times \mathrm{G})$. Anyway, there was a trend for higher yield stress with higher percentage of SCA and higher modulus with higher percentage of MMA. In fact, the best formulations were the ones with the highest levels of the second and third factors.

During immersion in the ISS, the already referred to WU of all samples allowed them to be plasticized. The decrease in yield stress and modulus was very dramatic but, on the other hand, the samples with higher amounts of MMA, that were brittle, became very ductile; indeed, no specimen fractured during the test. For the samples with higher amounts of AA, that were already ductile, swelling lead to strong decreases in the mechanical strength. As samples with higher MMA/AA molar ratio swelled much less, this factor was the main determinant of the mechanical properties after swelling (Table III). The SCA percentage had minor effects, and only when the MMA/AA ratio was at its lower level: as SCA sorbs less water then poly(acrylic acid) (PAA), it helped maintaining the mechanical properties, which were, in this case, higher for the upper level of the second factor.

To corroborate these qualitative discussion, statistical analysis of the experiments was performed with the "Statistica" ${ }^{\circledR} 5.0$ (StatSoft, USA) software. This was done in order to calculate the principal and interaction effects of each factor and to determine which of them mostly influenced the results. Table IV shows the results of this analysis. The higher the absolute value of the effect, the higher the influence of that factor (or interaction) on that property.

These values were plotted on normal probability plots to determine which of them were significant, and those which were, are shown on bold in Table IV. The main effect was the third one (molar ratio of the monomers), in agreement with that was previously previously discussed; SCA percentage and the interaction between the second and the third factor had significant (but always smaller) effects on some properties. The first factor (SCA particle size) had no significant influence on the analyzed properties; one possible reason for that could be the small range of variation of this factor.

Based on these data, it was decided to make another set of experiments in order to study the MMA/AA ratio on higher levels, since this was the most important factor and presented positive effects for all properties, except for WU/WL. The SCA particle size was chosen to vary on a larger range to determine if any possible effect of this factor was masked by the proximity of levels in the first factorial matrix. Finally, the SCA amount was kept fixed at $60 \%$, since it was intended to use the highest possible amount of biodegradable material and the results had shown that it had positive (or negligible) effects on all properties, with the exception of setting time. With these considerations, the matrix in Table II was constructed.

\subsection{Second matrix}

Table V and Figs. 4 and 5 show some of the properties measured for the compositions of the second matrix. The water uptake after seven days of immersion was again very dependent on the relative ratio of monomers, with only minor effects of the particle size: it seemed that with smaller particles the time to reach equilibrium was longer

T A B L E I V Values of each effect for the properties measured

\begin{tabular}{|c|c|c|c|c|c|c|c|}
\hline Effect* $*$ & $\mathrm{WU}_{7}$ & $\mathrm{WL}_{7}$ & $\sigma_{\mathrm{y}}$ & $E_{1 \%}$ & $\sigma_{\text {yas }}$ & $T_{\max }$ & $t_{\mathrm{s}}$ \\
\hline 1 & -0.10 & 0.09 & 12.50 & -0.20 & 0.00 & -9.00 & -0.35 \\
\hline 2 & 4.05 & -0.14 & 25.50 & 0.22 & 0.80 & -21.75 & -2.05 \\
\hline 3 & -18.69 & -4.37 & 12.50 & 0.97 & 7.45 & -42.75 & 4.60 \\
\hline 12 & 0.13 & 0.22 & 12.50 & -0.05 & 0.15 & 9.50 & 0.05 \\
\hline 13 & -0.39 & 0.23 & -3.50 & 0.30 & 0.10 & -1.50 & -0.10 \\
\hline 23 & -1.31 & 0.86 & 10.50 & -0.32 & -0.70 & -16.25 & -1.20 \\
\hline 123 & -0.73 & 0.04 & -4.50 & 0.15 & 0.15 & -8.00 & 0.10 \\
\hline
\end{tabular}

$* 1,2$, and 3 are the effects of each factor (see Table I); 12,13 and 23 are the effects of the interaction between 2 factors and 123 is the effect of the interaction between all of them. 
T A B L E V Formulations from the second factorial and their properties

\begin{tabular}{llllllll}
\hline Formulation & Levels & $\mathrm{WU}_{7}(\%)$ & $\mathrm{WL}_{7}(\%)$ & $\sigma_{\mathrm{y}}(\mathrm{MPa})$ & $E_{1 \%}(\mathrm{GPa})$ & $\sigma_{\mathrm{as}}(\mathrm{MPa})$ & $T_{\mathrm{max}}\left({ }^{\circ} \mathrm{C}\right)$ \\
\hline $\mathrm{I}$ & -+ & 27.5 & 3.80 & $64 \pm 2.6$ & $1.8 \pm 0.30$ & $17 \pm 1.2$ & 53 \\
$\mathrm{~J}$ & ++ & 28.0 & 4.55 & $65 \pm 4.1$ & $2.0 \pm 0.13$ & $13 \pm 1.2$ & 45 \\
$\mathrm{~K}$ & -- & 45.2 & 2.73 & $57 \pm 15$ & $2.1 \pm 0.26$ & $9.0 \pm 0.77$ & 73 \\
$\mathrm{~L}$ & +- & 42.3 & 3.93 & $62 \pm 11$ & $2.1 \pm 0.12$ & $7.1 \pm 0.40$ & 70 \\
\hline
\end{tabular}
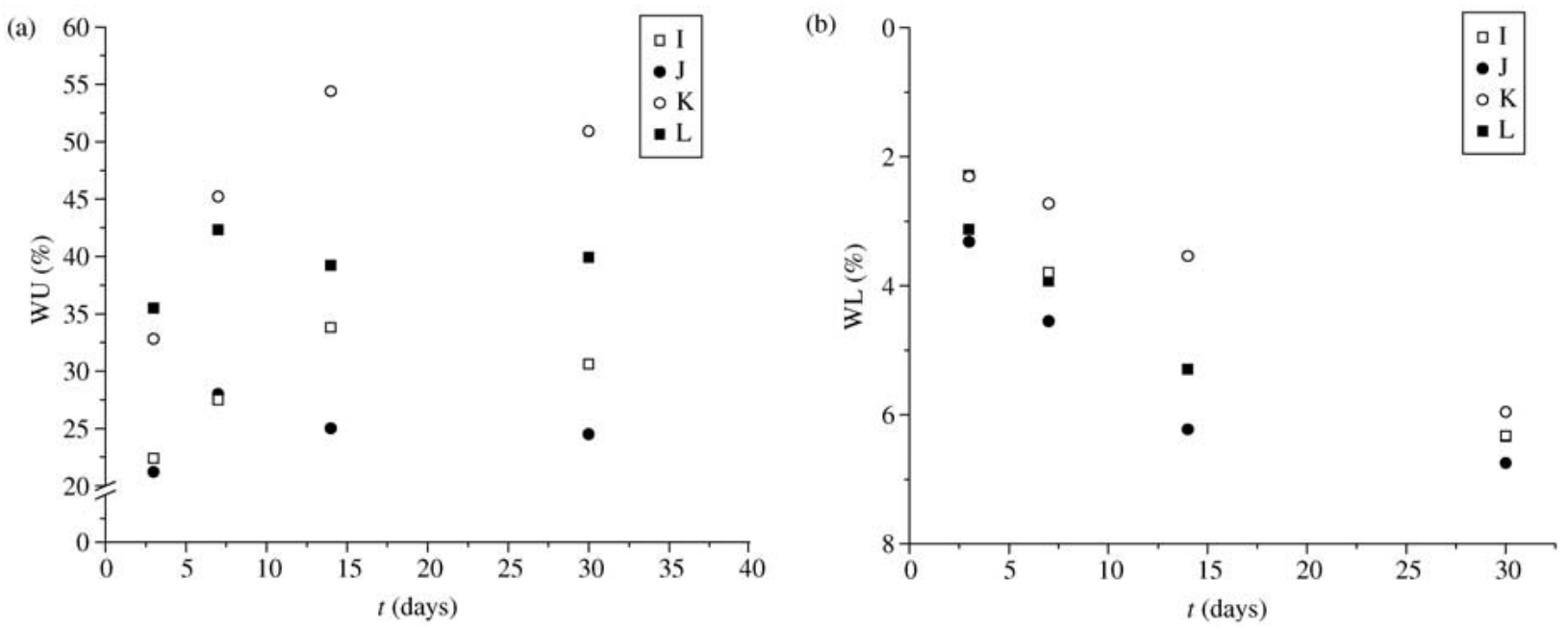

Figure 4 Swelling (a) and degradation (b) of selected samples from the second matrix. Formulations codes are indicated in Table V.

and the water uptake was higher for longer immersion times (30 days; see Fig. 4(a)). Regarding weight loss, a different behavior can be seen: the most important factor seemed to be the particle size, and it was remarkable that the formulations with higher WU presented lower WL, and vice-versa.

The mechanical behavior of the four formulations were very similar to the ones previously shown. As they contained high percentage of MMA, they were brittle after preparation, although they became more ductile after several days of storage in the controled $\mathrm{RH}$ chamber. There have been no significant differences between the four formulations, meaning that an increase in the MMA/AA ratio beyond a certain point does not increase the yield stress or $E_{1 \%}$. As expected, samples with higher amounts of MMA maintained better mechanical properties than the others after seven days of swelling in the ISS, and all of them were very ductile, as can be seen in Fig. 5 .

Finally, as for the trend found in the first factorial, the increase in the MMA amount decreased the $T_{\max }$ and increased the $t_{\mathrm{s}}$ of formulations I and $\mathrm{J}$ when compared to the other two, while the increase in SCA percentage $(60 \%$ against $55 \%$ in the samples from the first factorial) decreased the $t_{\mathrm{s}}$ (compare $\mathrm{K}$ with $\mathrm{C} / \mathrm{E}$ ).

The Statistica ${ }^{\circledR}$ software was applied to these data as well, and the results are on Table VI. After plotting on normal probability plots, it was found that there was no significant effect on the mechanical properties, while for the others the situation was similar to the one in the first matrix: only the MMA/AA ratio had significant effects, with no one coming from the SCA particle size.

It should be stressed herein that this analysis was done with data from seven days of swelling. As it is shown in Fig. 4(a), particle size seemed to have important effects (that were confirmed with the statistical analysis) for

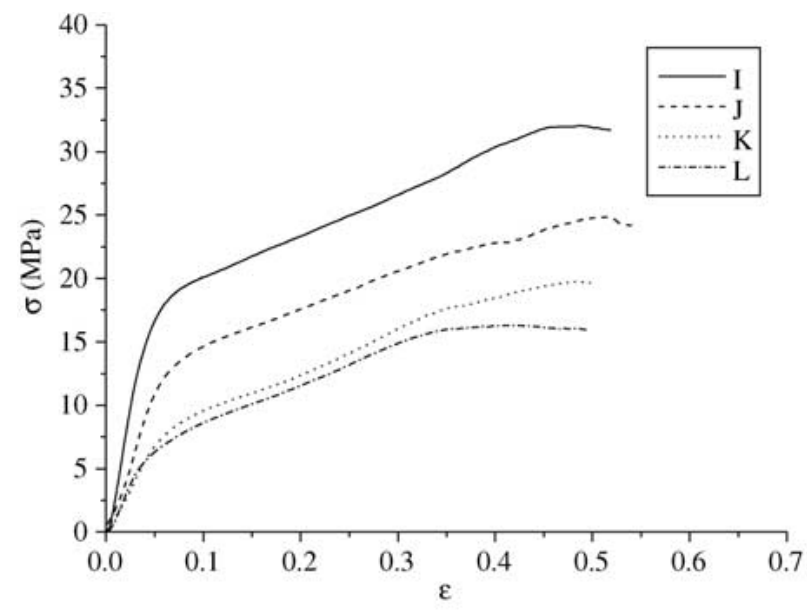

Figure 5 Compressive stress-strain curves of selected specimens after seven days of swelling in ISS. Formulations codes are indicated in Table V.

longer immersion times, and this could probably have implications in the long term mechanical behavior. This will be assessed in future works.

The combined results from the two factorial studies enabled us to draw some conclusions regarding the best formulations for further studies: (1) as the SCA particle size had practically no effect on any property (the only exception is the behavior depicted in Fig. 4), only the fraction lower than $125 \mu \mathrm{m}$ will be used, since it will allow for a better mixing with other components that would be also incorporated in future studies (such as bioactive glasses, antibiotics,); these components usually have a very fine granulometry; (2) since our intention was to use the maximum possible amount of SCA that yet allowed reasonable handling properties, it was decided to use a maximum of $60 \%$ of the polymer, since it was not possible to produce acceptable specimens with more than 
T A B L E V I Values of each effect for the properties measured

\begin{tabular}{|c|c|c|c|c|c|c|c|}
\hline Effect & $\mathrm{WU}_{7}$ & $\mathrm{WL}_{7}$ & $\sigma_{y}$ & $E_{1 \%}$ & $\sigma_{\text {yas }}$ & $T_{\max }$ & $t_{\mathrm{s}}$ \\
\hline 1 & 1.67 & 0.898 & 2.77 & 0.074 & -2.86 & -6 & 0.62 \\
\hline 2 & -15.90 & 0.852 & 4.87 & -0.26 & 6.62 & -26 & 2.5 \\
\hline 12 & 2.05 & -0.248 & -2.07 & 0.14 & -1.06 & -5.5 & 0.29 \\
\hline
\end{tabular}

this amount; (3) as the MMA/AA was the most important factor and changes in their value gave rise to quite different swelling and curing properties (what would have implications in their in vivo use) without significantly affecting the dry compressive properties, it was decided to use both levels of the second factorial in the following studies to assess how this ratio would affect other properties (tensile properties, bioactivity,).

\subsection{Comparison with the norm ASTM F-451 [17] and with Palacos ${ }^{\mathrm{B}} R$}

The minimum yield stress required by ASTM is $70 \mathrm{MPa}$. As it is shown in Tables III and V, compositions C, I, J, K and $\mathrm{L}$ would be accepted by the norm (at the $95 \%$ confidence level). Although there was no significant differences between any pair of them, compositions with highest amounts of SCA and MMA (I and J) had the smallest dispersion with high values of $\sigma_{y}$, maybe because of less effects of plasticization by ambient water, what is also an important parameter, since it is obviously the aim to obtain the minimum variability between specimens.

Regarding curing parameters, it should be recalled that the system used was not the one recommended by ASTM, so a direct comparison with values required by it could not be made. Anyway, measurements with Palacos ${ }^{\circledR} \mathrm{R}$ bone cement (the actual gold standard for clinical application) using our system gave results of $53{ }^{\circ} \mathrm{C}$ for $T_{\max }$ and a $t_{\mathrm{s}}$ of $13.5 \mathrm{~min}$. So, although formulations containing higher amounts of MMA presented acceptable curing temperature, all of them had very short setting times, which seems to be so far the main disadvantage of these cements. These characteristics will continue to be studied, and, by varying the amount of initiator and activator, we intend to reach values in the range specified by ASTM standart.

\subsection{Tensile properties of a selected formulation}

Table VII and Fig. 6 show the results from tensile experiments of a selected formulation. As it happened for compression tests, the mechanical strength and modulus had significant decreases, while ductility and toughness

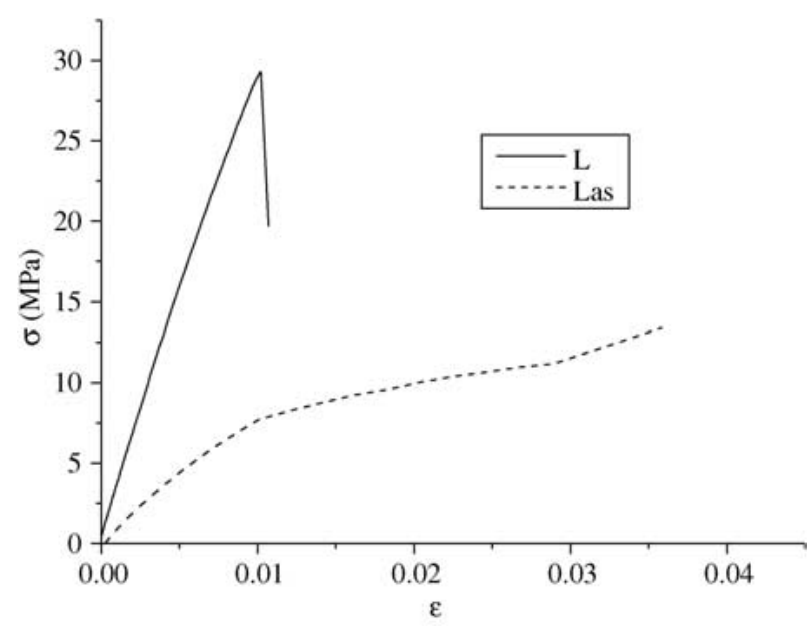

Figure 6 Tensile stress-strain curves of selected specimens from formulation L, both before (L) and after (Las) seven days of swelling in ISS. Formulations codes are indicated in Table V.

presented very large improvements. This is a very important positive characteristic of these formulations, since cements with lower modulus and creep resistance and higher ductility would be able to withstand large strains without fracture, distribute the load to the bone and allow the prosthesis to subside within the bone [18] It is also an advantage of these cements when compared to the conventional ones, which are brittle and do not show any improvement in mechanical properties after immersion.

This difference in the tensile behavior of dry and swelled states is clearly shown on the fracture surfaces of Fig. 7. One can see that after swelling the failure mode changed from a brittle one, with a smooth surface denoting rapid failure after the crack nucleation around a pore or an heterogeneity inside the specimen (Fig. 7(a)) to a more ductile one (Fig. 7(b)), with a very rough surface typical of fractures with low speed crack propagation; although pores continued to be crack generators, the plasticized matrix was able to deform much more prior to the failure.

A more detailed observation reveals other important characteristic of these cements: as prepared, the specimens are very dense and compact (with exception of a few macropores arising from inadequate preparation, see Fig. 7(c)) but after swelling they become very microporous, due to the leaching of un- or poorly polymerized monomers and/or plasticizers from the SCA (Fig. 7(d)). As already stated, this phenomenom, despite having deleterious effects on modulus and mechanical strength, could be very advantageous from the biological point of view since, with the addition of bioactive fillers, it could induce the growth of new bone inside the material, providing the adhesion to natural bone and, at

T A B L E V I I Tensile properties for two selected formulations

\begin{tabular}{lcllll}
\hline Formulation & UTS $(\mathrm{MPa})$ & $E_{1 \%}(\mathrm{GPa})$ & $\varepsilon_{\mathrm{f}}(\%)$ & $E_{\text {break }} * 100(\mathrm{~J})$ & $K(\mathrm{MPa})$ \\
\hline $\mathrm{L}$ & $24 \pm 0.74$ & $2.9 \pm 0.21$ & $1.3 \pm 0.25$ & $2.5 \pm 0.41$ & $0.14 \pm 0.027$ \\
$\mathrm{~L}$ (as) & $9.0 \pm 0.45$ & $0.49 \pm 0.11$ & $5.0 \pm 1.2$ & $5.4 \pm 1.0$ & $0.22 \pm 0.039$ \\
\hline
\end{tabular}

(as) $=$ after seven days of swelling in the isotonic saline solution; UTS $=$ ultimate tensile strength; $\varepsilon_{\mathrm{f}}=$ strain to failure; $E_{\mathrm{break}}=$ energy to fracture, $K=$ toughness. 

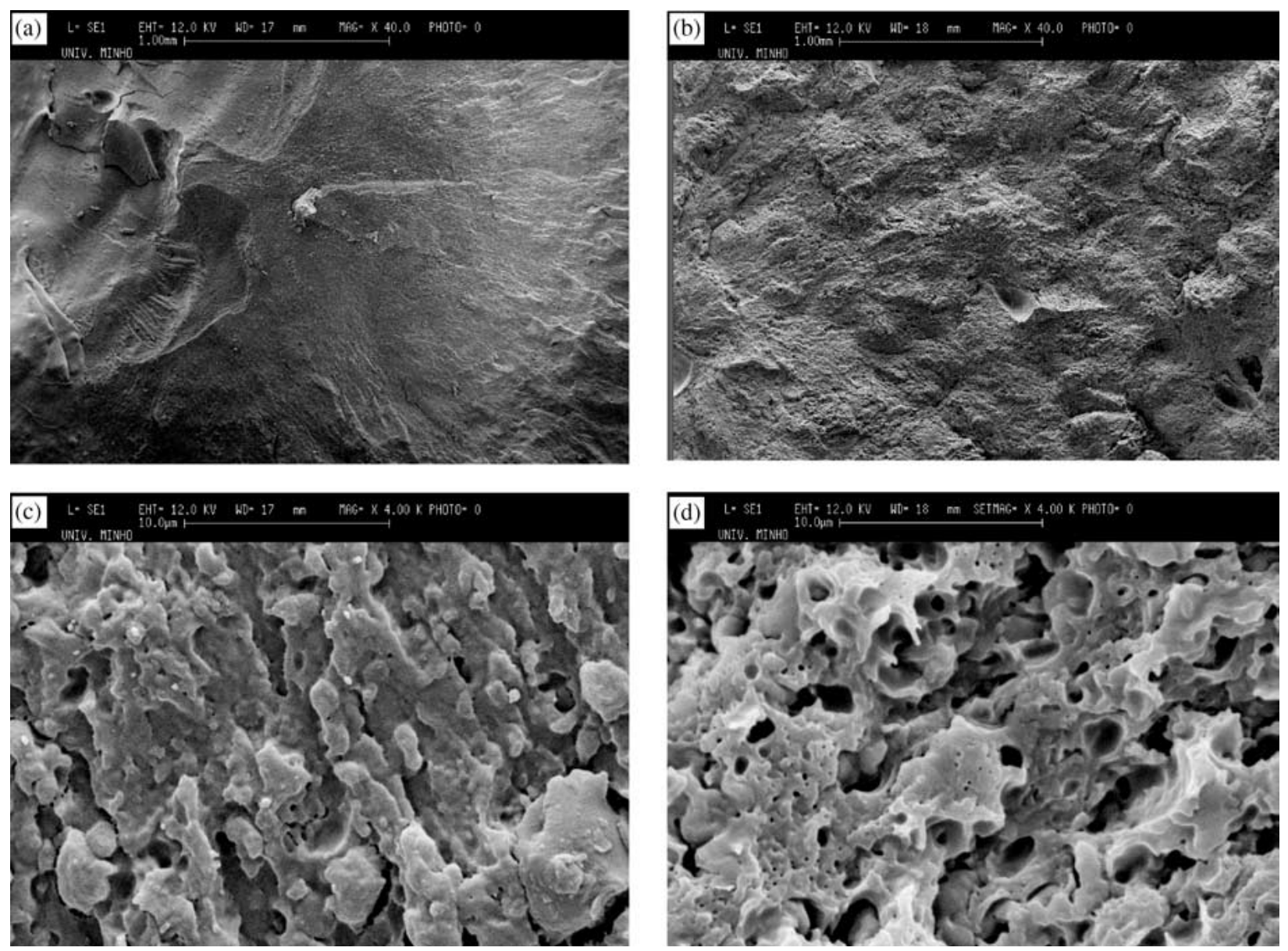

Figure 7 SEM microphotographs of surface fracture of specimens from formulation L. (a) and (c) as prepared; (b) and (d) after seven days of swelling in ISS. Formulations codes are indicated in Table V.

the same time, compensating for the decrease in the mechanical properties.

This evolution of mechanical and morphological behavior with immersion is being more extensively studied, using other formulations and a more expanded time scale, and will be published in a future paper.

\subsection{Mechanical results in wet environment}

Bone cements are always simultaneously subjected to cyclic and static loads. In the last case, the material will undergo a continuous, slow, long-term deformation under the applied stress, usually called creep. Such phenomena may be simulated and studied in in-vitro conditions. Fig. 8 shows the creep results obtained in specimens from samples $\mathrm{K}$ and $\mathrm{I}$, under the same mechanical/time program.

The typical creep behavior detected in polymeric systems is visible in the results: an "instantaneous", elastic response followed by a delayed elastic response (that may include an irreversible viscous component). Formulation I shows a smaller immediate elastic deformation and the viscoelastic strain is also less pronounced than K. Even in formulation I, that is the most stable in terms of creep deformation, it is obvious that the mechanical performance of such materials changes strongly with time, as may happen with other polymer-based biomaterials. Note that, in the viscoelastic linear regime, the representation of the creep compliance $D(t)=\varepsilon(t) / \sigma$, would be independent on $\sigma$,

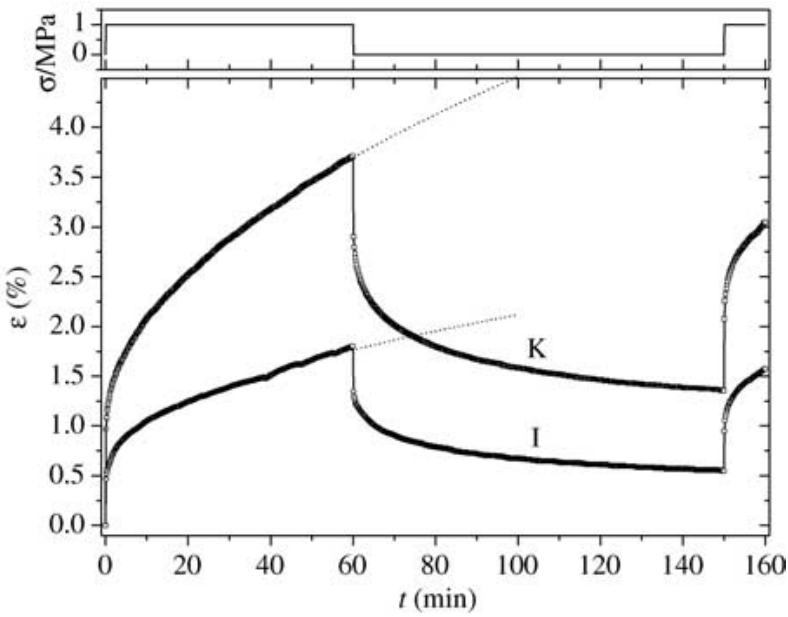

Figure 8 Creep/recovery data obtained in the wet state, at $37^{\circ} \mathrm{C}$. The stress program is shown in the top graphics whereas the recorded strain is shown in the bottom graphics, as a function of time. The dotted lines are the fitting of the creep tests using Equation 3; the adjusted parameters are in Table VIII. Formulations codes are indicated in Table V.

i.e. it would define a viscoelastic property only dependent on the material and temperature.

The results in Fig. 8 indicates that such kind of static mechanical tests, specially if the sample is immersed in simulated physiological solutions, may be of great importance when one pretends to characterize new biomaterials aimed to be used in load-bearing applications. 
T A B L E V I I I Adjustable parameters obtained from the fitting of the creep results in wet conditions (Fig. 8) with the hyperbolic sine model (Equation 3)

\begin{tabular}{lll}
\hline & $\mathrm{K}$ & $\mathrm{I}$ \\
\hline$\sigma_{a}(\mathrm{Mpa})$ & $1.16 \pm 0.01$ & $2.17 \pm 0.04$ \\
$N$ & $0.511 \pm 0.002$ & $0.475 \pm 0.006$ \\
$t_{0}^{-n} \cdot \operatorname{sin~} \mathrm{h}\left(1 / \sigma_{b}\right)$, with $\sigma_{b}(\mathrm{Mpa})$ & $0.337 \pm 0.003$ & $0.184 \pm 0.006$ \\
and $t_{0}(\mathrm{~min})$. & & \\
\hline
\end{tabular}

Obviously, the creep times exploited in the tests are well below the typical implantation time in current clinical applications. Therefore, it would be of interest to adapt models that permit to fit the results and predict the deformation behavior at higher time-scales. Many equations have been used for this purpose. One of the most used creep functions is the hyperbolic sine equation, the first which predicts the stress relaxation efforts [19]:

$$
\varepsilon(t)=\sinh \frac{\sigma}{\sigma_{a}}+\left(\frac{t}{t_{0}}\right)^{n} \sinh \frac{\sigma}{\sigma_{b}}
$$

where $\varepsilon$ and $\sigma$ are the strain and stress, and $\sigma_{a}, \sigma_{b}, n$ and $t_{0}$ are adjustable parameters. As commented by Turner [19], this equation combines the venerable power law for the time dependence with nonlinearity with respect to the stress.

The data in Fig. 8 were fitted with Equation 3 in the creep region (first $60 \mathrm{~min}$ ). As only one stress was analyzed for each sample $(\sigma=1 \mathrm{MPa})$, we will have a natural dependence between the $\sigma_{b}$ and $t_{0}$ parameters. Therefore only $\sigma_{a}$, and $n$ can be directly obtained. The adjusted parameters are shown in Table VIII. We should carry out experiments at different stresses in order to obtain the four parameters by fitting all the results. This is beyond the scope of this paper, that only pretends to suggest that the use of specific models may help in the prediction of long-term behavior of biomaterials under stress.

The fitting of the creep data with Equation 3 was carried out using a Levenberg-Marquard algorithm implemented in Origin ${ }^{\circledR} 6.0$ (Microcal Software, USA). A good convergence was found for both materials, in which the final values did not depend on the initial guesses.

The data in Fig. 8 also included the mechanical recovery experiments of the two samples $(\sigma=0)$, that were monitored during a time longer than the creep tests (90 min). After the instantaneous elastic recovery, the strain continues to decrease, but the results seem to suggest that probably a fraction of the deformation will not be recovered for a long time. This is an indication that an irreversible viscous component exists in the total rheological behavior of the materials. Such creep/ recovery experiments may be used to characterize this pure viscous part, with a viscosity $\eta$. For example, if we assume a simple viscous behavior, then $\eta=\sigma t / \varepsilon_{\infty}$, where $\sigma$ is the creep stress, applied during a time $t$ and $\varepsilon_{\infty}$ the reminiscent strain after an infinite time of recovery.

Fig. 8 also shows the results of a short second creep program applied after the recovery. Obviously, the strain

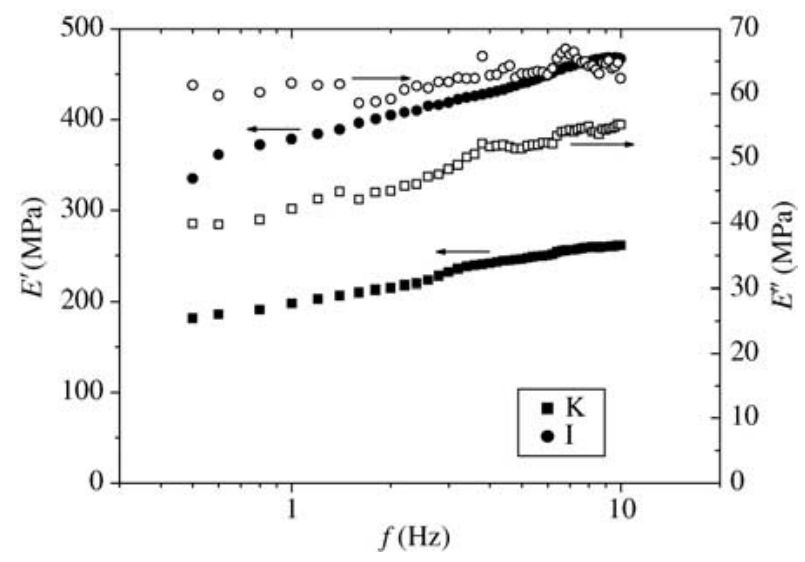

Figure 9 Frequency scan in $\mathrm{K}$ (squares) and I (circles). The experiments were carried out while the samples were immersed at $37^{\circ} \mathrm{C}$. Formulations codes are indicated in Table V.

will start at non-zero values (due to the prior noncomplete recovery). However, taking the origin of the strain values for the second creep experiments the ones measured at $t=150 \mathrm{~min}$ (the starting point of this test) it may be concluded that the first $10 \mathrm{~min}$ of both first and second creep runs give similar results, relatively of the elastic and viscoelastic components of creep. Such kind of experiments may be used to investigate any permanent changes that may occur in a material when subjected to a static load. In fact, if any modification takes place within the structure (e.g. degradation of the interface in heterogeneous materials induced by the mechanical stress) one should expect to see a different creep behavior in the second run.

Besides creep, the viscoelastic properties of the immersed samples were also analyzed by following the dynamic modulus while the frequency was scanned. The frequency ranged from 0.5 to $10 \mathrm{~Hz}$, that are of the order of magnitude of a masticatory function or of the equivalent frequency of the mechanical load felt by the bones under the typical movements of the patient. The storage and loss moduli are shown in Fig. 9. As expected the storage modulus $\left(E^{\prime}\right)$ tends to decrease as frequency decreases, because the molecular motions have enhanced time to proceed. The loss modulus $\left(E^{\prime \prime}\right)$ also follows the same trend of $E^{\prime}$, in such a way that the loss factor, $\tan \delta=E^{\prime \prime} / E^{\prime}$ is found to be almost independent on frequency in each material. For formulation $\mathrm{K}$ we obtained $\tan \delta \approx 0.21$ and, for formulation $\mathrm{I}$, $\tan \delta \approx 0.14$, i.e. although $\mathrm{K}$ shows a lower storage modulus than $\mathrm{I}$ it possesses a relative higher viscous component of the modulus. The $\tan \delta$ values obtained are rather high, conferring to the samples interesting damping properties. This will allow to absorb a fraction of the mechanical energy imposed to the material at relevant frequencies, which may be important for some biomedical application of such formulations, such as in orthopedic uses.

The storage modulus of I is typically above $350 \mathrm{MPa}$ which confers to this material a significant stiffness at $37^{\circ} \mathrm{C}$, taking into account that it absorbs around $30 \%$ of water. The lower storage modulus of $\mathrm{K}$ may be also responsible to the higher tendency to deform during creep (Fig. 8). In fact, the static and dynamic viscoelastic are related with each other; for example, the connection 
of the creep compliance $D(t)$ and the two components of the complex dynamic compliance, $D^{*}=1 / E^{*}=$ $D^{\prime}-\mathrm{i} D^{\prime \prime}$, is:

$$
D(t)=D_{\mathrm{g}}+\frac{2}{\pi} \int_{0}^{\infty}\left[\left(D^{\prime}-D_{\mathrm{g}}\right) / \omega\right] \sin \omega t d \omega+t / \eta_{0}
$$

$$
\begin{gathered}
D(t)=D_{\mathrm{g}}+\frac{2}{\pi} \int_{0}^{\infty}\left(D^{\prime \prime} / \omega-1 / \omega^{2} \eta_{0}\right) \\
(1-\cos \omega t) d \omega+t / \eta_{0}
\end{gathered}
$$

where $D_{g}$ is the glass-like compliance and $\eta_{0}$ is the steady flow viscosity [20]. The transposition between the creep and dynamic data is not an easy task and it is usually preferable to present these two quantities obtained experimentally, as it was done in this work.

\subsection{Dynamic mechanical thermal experiments in dry samples}

Traditionally, DMA experiments have been performed in dry samples, shaped to geometries that allow to characterize these viscoelastic properties in tensile, flexural, compression or shear modes. Such tests gained interest in the area of biomaterials because they provide both thermal and mechanical informations that can be correlated with the structure of the material and may give valuable indications on its clinical use [21]. In this work, DMA experiments on samples $\mathrm{K}$ and I were carried out in isochronal conditions (frequency of $1 \mathrm{~Hz}$ ).

Both storage and loss moduli are shown in Fig. 10, in the temperature range -5 to $90^{\circ} \mathrm{C}$. It can be seen that in the temperature range investigated both samples have similar viscoelastic properties. They change strongly when they are immersed in simulated fluids as it was investigated before. This is mainly due to the higher swelling ability of $\mathrm{K}$ and is a behavior similar to the one previously discussed for compression tests.

The peak in $E^{\prime \prime}$ at about $60^{\circ} \mathrm{C}$ is assigned to the glass transition of the copolymer formed from the liquid fraction. These values are below the glass transition of

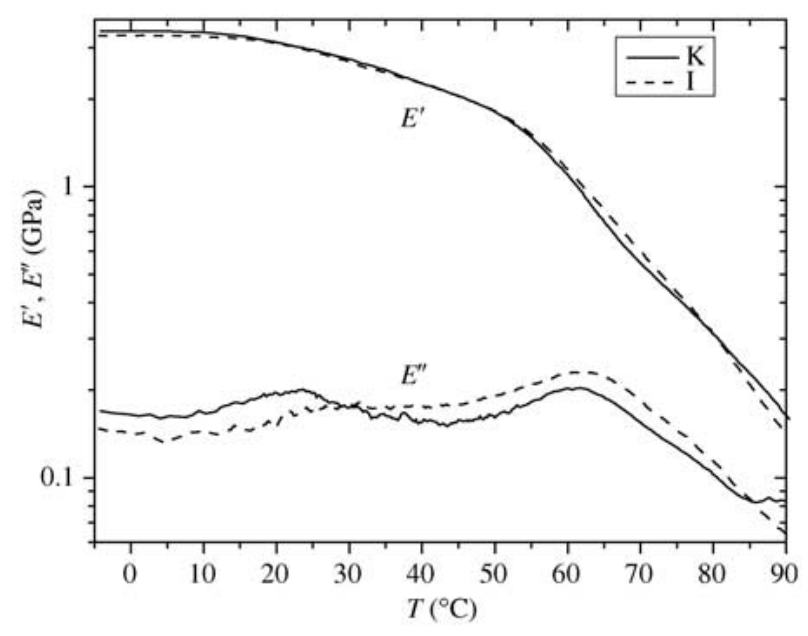

Figure 10 DMA results obtained in the dry state of $\mathrm{K}$ (solid lines) and I (dotted line) at $1 \mathrm{~Hz}$. Formulations codes are indicated in Table V. the pure homopolymer: $100{ }^{\circ} \mathrm{C}$ for PMMA and $106^{\circ} \mathrm{C}$ for PAA [16] and could be due to the strong plasticization effect of water in the acrylic acid units, due to the depletion of the hydrogen bonds. The same plasticization effect has been already seen in similar cement compositions [13].

\section{Conclusions}

The performed study allowed for the identification of parameters that most influence the results. In order of significance they were as follows: molar ratio of monomers, starch blend amount and starch blend particle size. It also enabled the selection of formulations with the best combination of mechanical, swelling/degradation and curing properties. The best formulations were the ones containing around $55-60 \%$ of SCA and $6-10 \%$ of AA. These combinations presented mechanical properties in the range of ASTM specifications and suitable polymerization temperatures.

The samples selected for preliminary tensile and DMA tests showed that it is possible to modulate the wet mechanical properties by varying the relative ratio of MMA/AA, although the mechanical properties in the dry state are similar (for high enough amounts of MMA). After swelling, the formulations could present characteristics such as increased toughness and creep and microporosity enhancement that, if appropriately balanced, would give rise to very interesting mechanical and biological performance in vivo. These materials also exhibit good damping properties in simulated physiological conditions.

The obtained results show that by means of optimizing their formulations it is possible to develop starch based partially degradable bone cements. These optimized materials disclose a combination of mechanical, viscoelastic, degradation, swelling and curing parameters that might allow for their successful introduction on clinical practice.

\section{Acknowledgments}

The authors acknowledges ICTP-CSIC (Spain) for providing the $\mathrm{DMOH}$ used in this study. L. F. B. also acknowledges Fundação Coordenação de Aperfeiçoamento de Pessoal do Ensino Superior (CAPES - Brasília, Brasil) for the PhD grant.

\section{References}

1. S. SHINZATO, T. NAKAMURA, T. KOKUBO and Y KitA MURA, J. Biomed. Mater. Res. 59 (2002) 225.

2. E. J. HARPER, M. BRADEN and W. J. BONFIELD, J. Mater. Sci. Mater. Med. 11 (2000) 491.

3. S. MORITA, K. FURUYA, K. ISHIHARA and N NAKA B AYA S HI, Biomaterials 19 (1998) 1601.

4. C. ELVIRA, B. VAÍZQUEZ, J. SAN ROMAÍN, B. LEVEnFELD, P. GINEBRA, X. GIL and J. A. PLANELl, $J$. Mater. Sci. Mater. Med. 9 (1998) 679.

5. M. E. GOMES, A. S. RIBEIRO, P. B. MALAFAYA, R. L. REIS and A. M. CUNHA, Biomaterials 22 (2001) 883.

6. M. E. GOMES, R. L. REIS, A. M. CUNHA, C. A BLITTERSWIJK and J. D. DE BRUIJN, ibid. 22 (2001) 1911.

7. M. E. GOMES, J. S. GODINHO, D. TCHALAMOV, A. M. CUNHA and R. L. REIS, Mater. Sci. Eng. C 20 (2002) 19. 
8. C. ELVIRA, J. F. MANO, J. SAN ROMAÍN and R. L. REIS, Biomaterials 23 (2002) 1955.

9. P. B. Malafaya, C. Elvira, A. GAllardo, J. SAN ROMAÍn and R. L. REIS, J. Biomater. Sci. Polym. Ed. 12 (2001) 1227.

10. R. A. SOUSA, G. KALAY, R. L. REIS, A. M. CUNHA and M. J. B E VIS, J. Appl. Polym. Sci. 77 (2000) 1303.

11. R. L. Reis, A. M. CUnha and M. J. BeVis, Med. Plast. Biomater. 4 (1997) 46.

12. C. S. PEREIRA, A. M. CUNHA, R. L. REIS, B. VAÍZQUEZ and J. SA N RoMAín, J. Mater. Sci. Mater. Med. 9 (1998) 825.

13. I. ESPigares, C. Elvira, J. F. MANo, B. VAízQuez, J. SAN ROMAN and R. L. REIS, Biomaterials 23 (2002) 1883.

14. D. C. MONTGOMERY, "Design and Analysis of Experiments", 3rd edn (John Wiley \& Sons, Singapore, 1991).

15. G. E. P. BOX, W. G. HUNTER and J. S. HUNTER, "Statistics for Experimenters"' (John Wiley \& Sons, New York, 1978).

16. J. BRANDRUP, E. H. IMMERGUT and E. A. GRULKE (eds),
'Polymer Handbook', 4th edn (Wiley Interscience, New York, 1999).

17. "Standard Specification for Acrylic Bone Cements. Annual Book of ASTM Standards, v.13.01, Specification F451-86" (ASTM, Philadelphia, 1986).

18. G. LEWIS, J. Biomed. Mater. Res. 38 (1997) 155.

19. S. TURNER, in "Encyclopedia of Materials Science and Technology", (Elsevier Science, Amsterdam, 2001).

20. J. D. FERRY, in "Viscoelastic Properties of Polymers", 3rd edn (John Wiley \& Sons, New York, 1980).

21. J. F. MANO, R. L. REIS and A. M. CUNHA, in "Polymer Based Systems on Tissue Engineering, Replacement and Regeneration", edited by R. L. REIS and D. COHN (Nato Science Series, Kluwer Academic, Dordrecht, 2002) pp. 139-164.

Received 02 December 2002

and accepted 09 July 2003 